\title{
Effect of vasopressin on hippocampal injury in a rodent model of asphyxial cardiopulmonary arrest
}

\author{
NAN ZHANG ${ }^{*}$, XIU-XIAN ZANG ${ }^{*}$, NING DONG, FANG LIU, SHAO-KUN WANG, \\ HE YAN, DA-HAI XU, XIAO-LIANG LIU and LI PANG \\ Department of Emergency Medicine, The First Hospital of Jilin University, Changchun, Jilin 130021, P.R. China
}

Received November 27, 2014; Accepted December 30, 2015

DOI: $10.3892 /$ etm.2016.3028

\begin{abstract}
The effect of vasopressin on the neuronal injury following the restoration of spontaneous circulation (ROSC) in cardiac arrest (CA) is not yet fully understood. The present study was conducted in order to investigate the effect of vasopressin alone, or in combination with epinephrine, on the ROSC and hippocampal injury in a rat model of asphyxial CA. Asphyxial CA was induced in 144 rats by clamping the tracheal tube, and animals were allocated equally into the following three groups: Treatment with vasopressin $(0.8 \mathrm{U} / \mathrm{kg})$; epinephrine $(0.2 \mathrm{mg} / \mathrm{kg})$; or vasopressin $(0.8 \mathrm{U} / \mathrm{kg})$ plus epinephrine $(0.2 \mathrm{mg} / \mathrm{kg})$. An additional 48 rats underwent a sham surgical procedure without asphyxial CA and cardiopulmonary resuscitation. Hippocampal tissue was harvested at 1, 3, 6 and $12 \mathrm{~h}$ post-ROSC, and the levels of p38 mitogen-activated protein kinase (MAPK) and nuclear factor $-\kappa \mathrm{B}(\mathrm{NF}-\kappa \mathrm{B})$ p65 were determined using immunohistochemistry. In comparison with rats treated with epinephrine alone, higher ROSC success rates were observed in rats treated with vasopressin, or vasopressin plus epinephrine. In addition, treatment with vasopressin attenuated hippocampal injury and reduced hippocampal p38 MAPK and NF- $\kappa$ B expression more efficiently compared with epinephrine alone. In conclusion, treatment with vasopressin exhibits a protective effect in patients experiencing CA, and this may be attributed to the inhibition of p38 MAPK and $\mathrm{NF}-\kappa \mathrm{B}$ expression.
\end{abstract}

\footnotetext{
Correspondence to: Professor Xiao-Liang Liu or Dr Li Pang, Department of Emergency Medicine, The First Hospital of Jilin University, 71 Xinmin Street, Changchun, Jilin 130021, P.R. China E-mail: 466634002@qq.com

E-mail: pangli211@163.com

*Contributed equally
}

Key words: asphyxial cardiopulmonary resuscitation, vasopressin, epinephrine, p38 mitogen-activated protein kinase, nuclear factor- $\kappa \mathrm{B}$

\section{Introduction}

Cardiopulmonary arrest (CA) is a major health concern that has a poor prognosis $(1,2)$. Although rigorous emergency procedures and cardiopulmonary resuscitation (CPR) have decreased the mortality rates of CA, long-term ( $\geq 12$ month) survival following the restoration of spontaneous circulation (ROSC) remains low $(3,4)$. Neurological damage is a common problem that adversely affects patients following CA and ROSC, and results in limited long-term survival. Although the rates of neurological disability among CA survivors have decreased from 32.9 to $28.1 \%$ between the years 2002 and 2009 (5), there are no specific therapies available to alleviate CA-associated brain injury.

The mechanisms underlying brain injury following CA remain poorly understood. Mitogen-activated protein kinases (MAPKs) are a family of signal transduction proteins, including extracellular signal regulated kinase and p38-MAPK, that are activated by cellular stresses (6). Sustained expression of $\mathrm{p} 38$ MAPK is associated with neuronal death and apoptosis (7), whereas the inhibition of p38 MAPK is neuroprotective in cerebral focal ischemia (8). As a result of these neuroprotective effects, p38 MAPK inhibitors have been tested as therapeutic agents for neural diseases (9).

Nuclear factor- $\kappa \mathrm{B}(\mathrm{NF}-\kappa \mathrm{B})$ is a transcription factor expressed throughout the nervous system (10). In response to ischemia, NF- $\kappa \mathrm{B}$ expression may promote cell death through apoptosis and necrosis (11). The inhibition of NF- $\mathrm{KB}$ may be able to reduce brain injury in rat models of middle cerebral artery occlusion (12) and hypoxic-ischemic brain damage (13). These results suggest that therapies targeting $\mathrm{NF}-\kappa \mathrm{B}$ could reduce brain injury following $\mathrm{CA}$ and subsequent CPR (14).

Pharmacological intervention, typically using epinephrine, is essential in the management of CA (15). Previous studies have demonstrated that epinephrine has a neuroprotective effect following CA; however, the use of epinephrine for this purpose remains controversial $(16,17)$. Epinephrine has a number of unfavorable side effects, such as long-term hypotension and ventricular dysrhythmia; therefore, there is a requirement for alternative therapies for $\mathrm{CA}$-associated brain injury (18).

Vasopressin is a peptide hormone that functions as a potent vasoconstrictor (19). Previous laboratory studies have indicated 
that a combination therapy of vasopressin and epinephrine improves histopathological outcome and cerebral blood flow more successfully compared to treatment with epinephrine alone (20-22). However, these studies have not investigated whether vasopressin alone or combined with epinephrine is able to reduce hippocampal injury. In the present study, a rodent model of asphyxial cardiac arrest is used to compare the effects of vasopressin and epinephrine alone, or in combination, on hippocampal injury following ROSC and the success rate of resuscitation.

\section{Materials and methods}

Animals and reagents. The Animal Care and Use Committee of Jilin University (Changchun, China) approved the experimental procedures performed in the present study. A total of 192 adult male Sprague-Dawley rats (weight, 270 20 g; age, 8 weeks) were purchased from the Experimental Animal Center at Jilin University and housed with ad libitum access to food and water under conditions of $20 \pm 2^{\circ} \mathrm{C}, 55-60 \%$ humidity and a 12-h light/dark cycle. Polyclonal anti-p38 MAPK and anti-NF- $\kappa$ B p65 antibodies were purchased from Beijing Boosen Biological Technology Co., Ltd. (Beijing, China). Vasopressin was purchased from Sigma-Aldrich (St. Louis, MO, USA) and epinephrine was purchased from Shanghai Harvest Pharmaceutical Co., Ltd. (Shanghai, China).

Surgical preparation of rats. The surgical procedure for asphyxial CA induction was performed as described previously (23). Briefly, Sprague-Dawley rats were anesthetized by intraperitoneal injection with $10 \%$ chloral hydrate (0.03 ml/g; Shanghai Harvest Pharmaceutical Co., Ltd.). Electrocardiographic monitoring was performed using limb leads (II) to measure the heart rate. A tracheotomy was performed, followed by intubation with an 18-gauge angiocatheter and mechanical ventilation using an animal ventilator (DW-2000-type; Shanghai Jiapeng Technology Co., Ltd., Shanghai, China). Catheterization of the femoral vein was performed to administer sodium heparin $(5 \mathrm{IU} / \mathrm{ml}$; Benny Biochemical Pharmaceutical Co., Ltd., Changzhou, China).

Induction of asphyxial cardiac arrest in experimental rats. Following a 10-min equilibration period after the operation, cardiac arrest was induced by clamping the tracheal tube for $5 \mathrm{~min}$. Cardiac arrest was confirmed by the loss of aortic pulsations, defined as a mean arterial blood pressure $<10 \mathrm{mmHg}$ (24). A total of 144 rats undergoing asphyxial CA and subsequent CPR were randomly allocated into three equally sized treatment groups: Rats treated with vasopressin $(0.8 \mathrm{U} / \mathrm{kg})$; epinephrine $(0.2 \mathrm{mg} / \mathrm{kg})$; or vasopressin $(0.8 \mathrm{U} / \mathrm{kg})$ plus epinephrine $(0.2 \mathrm{mg} / \mathrm{kg})$. An additional 48 rats underwent a sham surgical procedure without $\mathrm{CA}$ induction or CPR. After $10 \mathrm{~min}$ at room temperature, cardiac compression was performed manually at a rate of 180 compressions/min over the chest, with sufficient compression force to achieve $1 / 3$ of the anteroposterior chest diameter. The indicated drugs were administered once CPR began. Ventilation was commenced using $100 \%$ oxygen at a breathing rate of 70 breaths/min, with a tidal volume of $6 \mathrm{ml} / \mathrm{kg}$ and an exhale to inhale ratio of 1:1.5.
Assessment of ROSC. ROSC was evaluated by two independent observers. ROSC was indicated by the emergence of supraventricular rhythm detected by the electrocardiogram monitor (78354C; Hewlett Packard Enterprise, Palo Alto, CA, USA) and a mean arterial blood pressure of $\geq 20 \mathrm{mmHg}$ for $5 \mathrm{~min}$ (25). Following the administration of each drug, the number of successful ROSC cases and the length of time between CPR and ROSC was recorded. If animals did not achieve ROSC after 10 min of CPR, resuscitation was discontinued.

Microscopic analysis in the hippocampus. Following anesthetization by intraperitoneal injection with $30 \mathrm{mg} / \mathrm{kg}$ pentobarbital sodium (H. Lundbeck A/S, Valby Denmark), the rats were sacrificed by decapitation. Tissue from the hippocampal CA1 region was harvested at 1, 3, 6 and $12 \mathrm{~h}$ after ROSC. At each time point, 12 rats were sacrificed. Hematoxylin-eosin (HE) staining (Beyotime Institute of Biotechnology, Shanghai, China) was performed according to standard protocols and the tissue was evaluated by two independent investigators using a light microscope (JEM-1200EX; Sweden).

Ultrastructural analysis of hippocampal cells. For ultrastructural analysis, hippocampal CA1 tissue samples (size, $\sim 2 \times 1 \times 1 \mathrm{~mm}$ ) were fixed with $2.5 \%$ glutaraldehyde (Sigma-Aldrich) and embedded in EPON resin (Hexion Inc., Columbus, $\mathrm{OH}, \mathrm{USA})$. Ultra-thin (50 nm) sections were cut using an ultra microtome (LKB8800III; LKB Vertriebs $\mathrm{GmbH}$, Vienna, Austria) and stained with uranyl acetate (Shanghai Yanjing Biological Technology Co., Ltd., Shanghai, China). An independent observer analyzed each sample by transmission electron microscopy (JEM-1200EX; Japan Electron Optics Laboratory Co., Ltd., Tokyo, Japan).

P38 MAPK and NF- $\kappa$ B p65 expression levels. The expression levels of p38 MAPK and NF- $\kappa$ B p65 were detected using immunohistochemistry. Briefly, 4- $\mu \mathrm{m}$ sections were fixed in $5 \%$ formaldehyde for 7 days and dehydrated with decreasing concentrations of ethanol. Paraffin sections were autoclaved (C16S01; Supor Co., Ltd., Hangzhou, China) at $98^{\circ} \mathrm{C}$ in citrate buffer (pH 6.0; Shanghai Meilian Biological Institute, Shanghai, China) for $10 \mathrm{~min}$. Sections were transferred to glass slides and treated with $3 \%$ hydrogen peroxide for $15 \mathrm{~min}$ in order to inactivate endogenous peroxides. The sections were then blocked using $1 \%$ goat serum (Beyotime Institute of Biotechnology) in phosphate-buffered saline (PBS) for $15 \mathrm{~min}$ at room temperature, then incubated with rabbit anti-human p38 MAPK (1:200; bs-0637R; Beijing Boosen Biological Technology Co., Ltd.) or NF-kB p65 (1:200; bs-3543R; Beijing Boosen Biological Technology Co., Ltd.) polyclonal antibodies in $1 \%$ goat serum for $12 \mathrm{~h}$ at $4^{\circ} \mathrm{C}$. Following antibody incubation, slides were incubated with biotin-conjugated mouse anti-rabbit IgG (1:500; bs-0296P-Bio; Beijing Boosen Biological Technology Co., Ltd.) for $10 \mathrm{~min}$ at $37^{\circ} \mathrm{C}$. Slides were then washed with PBS three times, incubated with horseradish peroxidase (labeled with streptavidin; Beyotime Institute of Biotechnology) for $10 \mathrm{~min}$ at $37^{\circ} \mathrm{C}$, and incubated with 3,3'-diaminobenzidine (Maixin Biotechnology Co., Ltd., Fuzhou, China) for 1-2 min. The slides were stained with hematoxylin, and stained tissues 
Table I. Staining score criteria for immunohistochemistry.

Positive cell number (score: $\%$ ) Intensity of staining score

Total score (degree of positive expression)

$\begin{array}{lll}0 & 0: \text { No color } & 0 \\ 1: \leq 25 & 1: \text { Faint yellow } & 2-3(+) \\ 2: 26-50 & 2: \text { Pale brown } & 4-5(++) \\ 3: 50-75 & 3: \text { Brown } & 6-7(+++) \\ 4:>75 & & \end{array}$

Total Score $=$ Positive cell number score + Intensity of staining score .

Table II. Baseline characteristics of the rats.

\begin{tabular}{|c|c|c|c|c|}
\hline Group & Body weight (g) & Heart rate (beats/min) & $\mathrm{SBP}(\mathrm{mmHg})$ & $\mathrm{DBP}(\mathrm{mmHg})$ \\
\hline Sham control & $267.13 \pm 21.68$ & $298 \pm 25.82$ & $125.13 \pm 8.95$ & $94.40 \pm 4.82$ \\
\hline Epinephrine & $270.00 \pm 17.57$ & $301 \pm 17.57$ & $131.00 \pm 10.00$ & $94.13 \pm 5.19$ \\
\hline Vasopressin & $268.53 \pm 19.37$ & $289 \pm 15.89$ & $129.00 \pm 8.98$ & $90.00 \pm 7.31$ \\
\hline Vasopressin + epinephrine & $270.40 \pm 17.23$ & $303 \pm 19.23$ & $127.00 \pm 9.69$ & $97.43 \pm 5.54$ \\
\hline
\end{tabular}

Data are presented as the mean \pm standard deviation. SBP, systolic blood pressure; DBP, diastolic blood pressure.

Table III. Comparison of ROSC.

\begin{tabular}{lccc} 
Group & & & $\begin{array}{c}\text { Number of times administered } \\
\text { during ROSC }\end{array}$ \\
\hline Sham control & ROSC success rate & ROSC time (sec) & N/A \\
Epinephrine & $48 / 48$ & $262.00 \pm 17.89$ & $2.40 \pm 0.89$ \\
Vasopressin & $24 / 48$ & $162.00 \pm 11.49^{\mathrm{a}}$ & $1.56 \pm 0.73$ \\
Vasopressin + epinephrine & $39 / 48^{\mathrm{a}}$ & $141.27 \pm 6.59^{\mathrm{a}, \mathrm{b}}$ & $1.36 \pm 0.50^{\mathrm{a}}$ \\
\hline
\end{tabular}

Data are presented as the mean \pm standard deviation or observed frequencies. ROSC, restoration of spontaneous circulation. ${ }^{\mathrm{a} P}<0.05 \mathrm{vs}$. epinephrine group; ${ }^{\mathrm{P}}<0.05$ vs. vasopressin group.

were analyzed by light microscopy (JEM-1200EX). Staining was assessed in 100 randomly selected cells under 10 fields in order to determine the staining intensity and the percentage of positive cells. Overall staining was measured using the immunoreactive score (IRS) that is calculated as a product of the intensity and percentage scores (26). Based on IRS, the staining was categorized as negative (IRS, 0), weak (IRS, 2-3), moderate (IRS, 4-5), and strong (IRS, 6-7). The staining score criteria are detailed in Table I.

Statistical analysis. Continuous variables were presented as the mean \pm standard deviation and categorical variables were expressed as observed frequencies. Continuous variables were analyzed using one-way analysis of variance and the Student-Newman-Keuls multiple comparisons test, and categorical variables were compared using the Fisher's exact test. Statistical analysis was performed using SPSS version 16.0 software (SPSS, Inc., Chicago, IL, USA), and $\mathrm{P}<0.05$ was considered to indicate a statistically significant difference.

\section{Results}

Comparison of ROSC success rate. Prior to the induction of asphyxial CA, no significant differences in the baseline characteristics were observed among the four groups (Table II). As presented in Table III, the success rate of ROSC in rats treated with vasopressin (39/48 rats), or with vasopressin plus epinephrine (42/48 rats), was significantly higher compared with rats treated with epinephrine alone $(24 / 48$ rats; $\mathrm{P}<0.05)$. In addition, the time required to achieve ROSC following treatment with vasopressin, or with vasopressin plus epinephrine, was significantly reduced compared to the rats treated with epinephrine alone $(\mathrm{P}<0.05)$. In addition, the administration number during ROSC following treatment with vasopressin plus epinephrine was significantly reduced compared with epinephrine alone $(\mathrm{P}<0.05)$.

Histological analysis of the hippocampus. In the hippocampus of sham control rats, normal neurons free of edema 

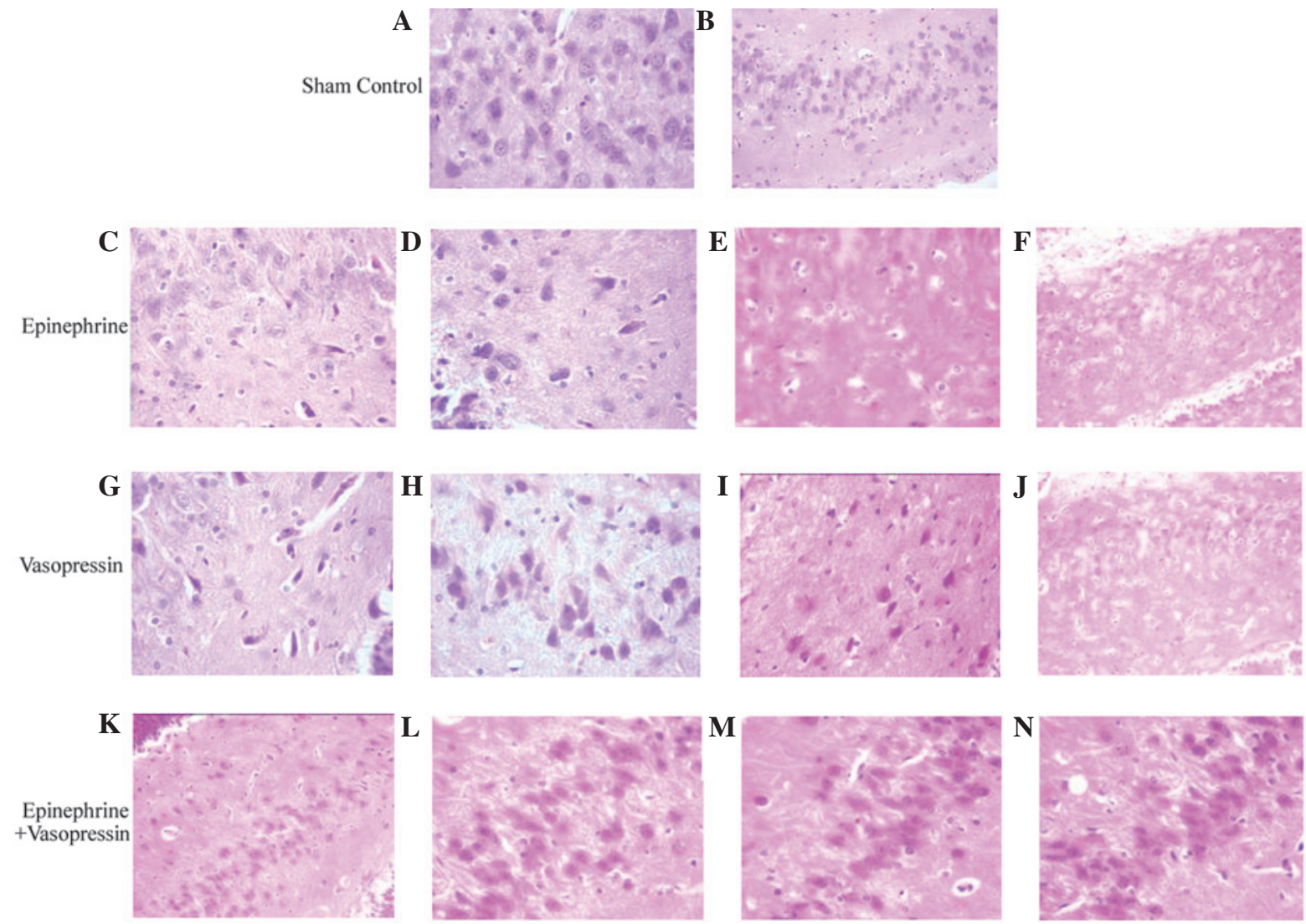

Figure 1. Histological changes of hippocampal CA1 region following asphyxial cardiac arrest. (A and B) Hematoxylin and eosin (HE) stained pathological sections of hippocampus in the sham control group presented with normal neurons without edema. (C-F) HE stained hippocampal tissue from rats treated with epinephrine presented with ambiguous or invisible nuclei, cytoplasmic cavity and aggravated evident edema of hippocampal tissue from rats sacrificed at (C) 1, (D) 3, (E) 6 and (F) $12 \mathrm{~h}$ following restoration of spontaneous circulation (ROSC). (G-J) HE stained hippocampal tissue from rats treated with vasopressin presented with ambiguous or invisible nuclei, and aggravated cytoplasmic cavity was detected [G-I, x400 magnification of hippocampal tissue from rats sacrificed at $(\mathrm{G}) 1$, (H) 3, (I) 6 and (J) $12 \mathrm{~h}$ after ROSC. (K-N) HE stained hippocampal tissue from rats treated with vasopressin plus epinephrine. Edema was not evident and the nucleus was ambiguous. Hippocampal tissue at (K) 1, (L) 3, (M) 6 and (N) 12 h after ROSC (magnification: A, C-E, G-I and L-N, x400; B, F, J and K, x200).

were observed (Fig. 1A and B). In contrast, all rats subjected to asphyxial CA displayed hippocampal neurons with ambiguous or invisible nuclei, cytoplasmic cavities and neural edema (Fig. 1C-J). At $1 \mathrm{~h}$ post-RPSC, edema was not detected in the hippocampal neurons of rats treated with a combination of vasopressin and epinephrine (Fig. 1K-N).

Ultrastructural changes of hippocampal neurons. As presented in Fig. 2A, normal structures of the hippocampal neurons were observed in the sham control group, while ultrastructural abnormalities within the neurons of the hippocampus were observed following asphyxial CA in rats treated with epinephrine (Fig. 2B and C). Asphyxial CA induced prominent mitochondrial defects, including swollen mitochondria and loss of the typical mitochondrial morphology (Fig. 2B). A loss of rough endoplasmic reticulum and mitochondrial fragmentation was also observed in neurons following asphyxial CA (Fig. 2C). These ultrastructural defects were attenuated by treatment with vasopressin or vasopressin plus epinephrine (Fig. 2D-G).

Induction of 38 MAPK expression by asphyxial CA. A small quantity of p38 MAPK was detectable in the hippocampal neurons of the sham control group; however, p38 MAPK was abundant in all of the rats following asphyxial CA (Fig. 3A-D). Quantification of the staining indicated that the expression level of p38 MAPK was significantly higher following asphyxial CA (Fig. 3E; P<0.05). However, the expression level of p38 MAPK was significantly reduced in rats treated with vasopressin, or vasopressin plus epinephrine, in comparison with rats treated with epinephrine alone $(\mathrm{P}<0.05)$. No significant difference was observed between the expression levels of p38 MAPK in the rats treated with vasopressin plus epinephrine compared with vasopressin alone $(\mathrm{P}>0.05)$.

$N F-\kappa B$ p65 expression following asphyxial $C A$ and treatment with vasopressin. Very low expression levels of NF- $\mathrm{B}$ p65 were detected in the sham control group, while abundant NF- $\kappa \mathrm{B}$ p65 staining was detected following asphyxial CA (Fig. 4A-D). Positive staining scores of NF- $\kappa$ B p65 were significantly higher in each asphyxial CA group in comparison with the sham control group (Fig. 4E; $\mathrm{P}<0.05$ ). As observed in the MAPK analyses, $\mathrm{NF}-\kappa \mathrm{B}$ p65 staining was significantly reduced in rats treated with vasopressin, or vasopressin plus epinephrine, in comparison with those treated with epinephrine alone $(\mathrm{P}<0.05)$. There were no significant differences between the synergistic effects from combining vasopressin and epinephrine. 

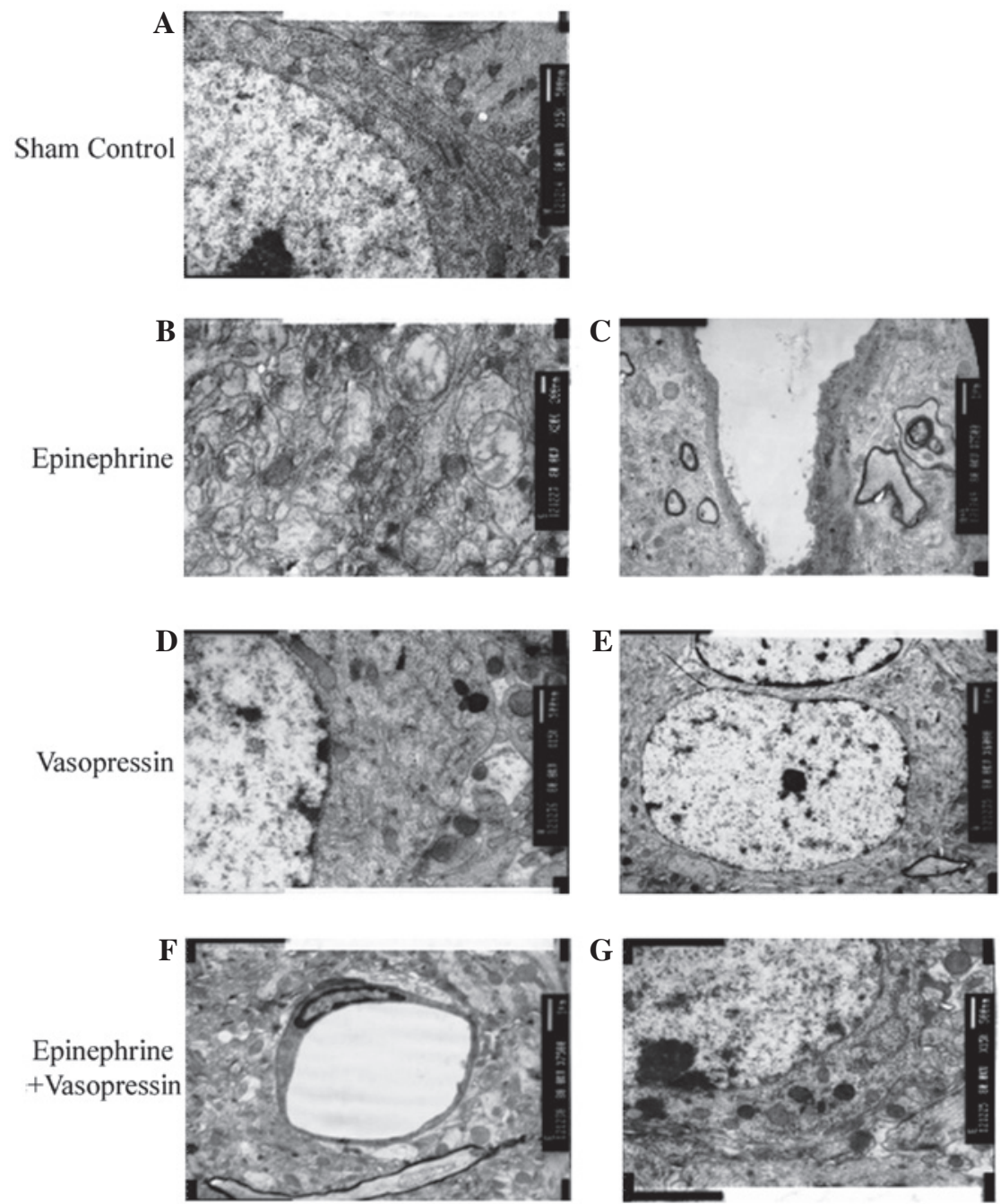

Figure 2. Ultrastructural analysis of the hippocampus CA1 region following asphyxial cardiac arrest (magnification: x8,000). (A) In the sham control group, large quantities of rough endoplasmic reticulum, round mitochondria and clear intramitochondrial ridges are observed. (B and C) In the epinephrine treated group, (B) swollen mitochondria with focal breakdown of the intramitochondrial ridge, and membrane intrusion into the mitochondrial cavity is observed; (C) slightly thickened capillary vessels, incomplete or partially disappeared membrane structures, and disarrangement of the myelin sheath is also observed. (D and E) In the vasopressin treatment group, rough endoplasmic reticulum, free ribosomes, round or oval-shaped mitochondria with diminished intramitochondrial ridges and lysosomes are observed. (F and G) Ultrastructure of the hippocampus in the vasopressin plus epinephrine treated group presents with (F) rough endoplasmic reticulum, free ribosomes, small rounded-shaped mitochondria, compact ridges, increased lysosomes and a small quantity of lipofuscin granules. (G) Flat capillary endothelial cells, thin and intact membrane structures, and minimal protrusions into the mitochondrial cavity are also observed.

\section{Discussion}

The present study demonstrated that treatment with vasopressin following CA improved the chance of survival and attenuated ultrastructural changes associated with hippocampal injury. In addition, the expression of p38 MAPK and $\mathrm{NF}-\kappa \mathrm{B}$ p65 was significantly reduced in the hippocampus of rats treated with vasopressin, as compared with those treated with epinephrine. Furthermore, combination therapy of vasopressin and epinephrine appeared to have a synergistic effect in attenuating hippocampal injury; however, they did not induce these effects using the presently investigated mechanisms.

Animal studies have demonstrated that CA and CPR are able to promote injury in selectively vulnerable zones of the brain, including the hippocampus (27). The present study demonstrated that simultaneous administration of vasopressin and epinephrine during CPR improved the histopathological outcome following ROSC. These results are consistent with a previous study, which demonstrated that combination therapy with epinephrine and vasopressin improved the histopathological outcome, as compared with epinephrine alone (22).

Ultrastructural analyses in the present study demonstrated that vasopressin alone, or in combination with epinephrine, reduced edema and mitochondrial damage in hippocampal neurons. These beneficial results may have been due to the high cerebral blood flow induced by vasopressin (28). In addition to reducing hippocampal injury, combination therapy with vasopressin and epinephrine may permit lower doses of epinephrine, thereby minimizing adverse side effects.

p38 MAPK is activated following cerebral ischemia and contributes to ischemic/hypoxic neuronal cell death $(29,30)$. In the present study, immunohistochemistry demonstrated that p38 MAPK expression levels were significantly elevated for up to $12 \mathrm{~h}$ post-ROSC, thus suggesting that p38 MAPK 
$\mathbf{A}$

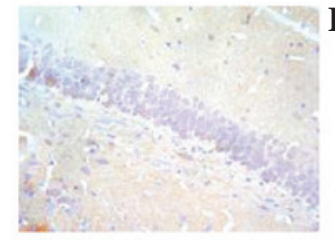

C
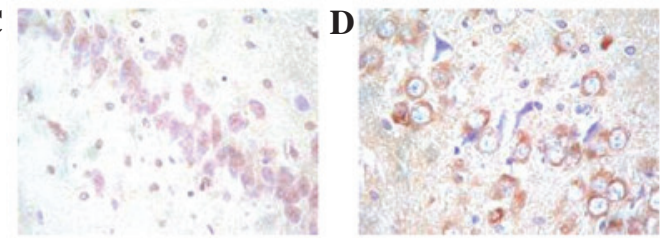

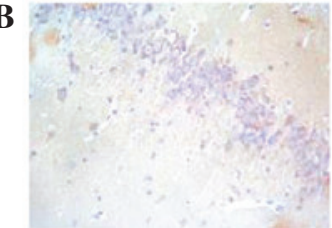

$\mathbf{E}$
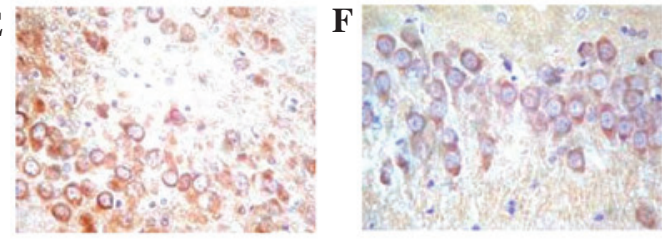

G
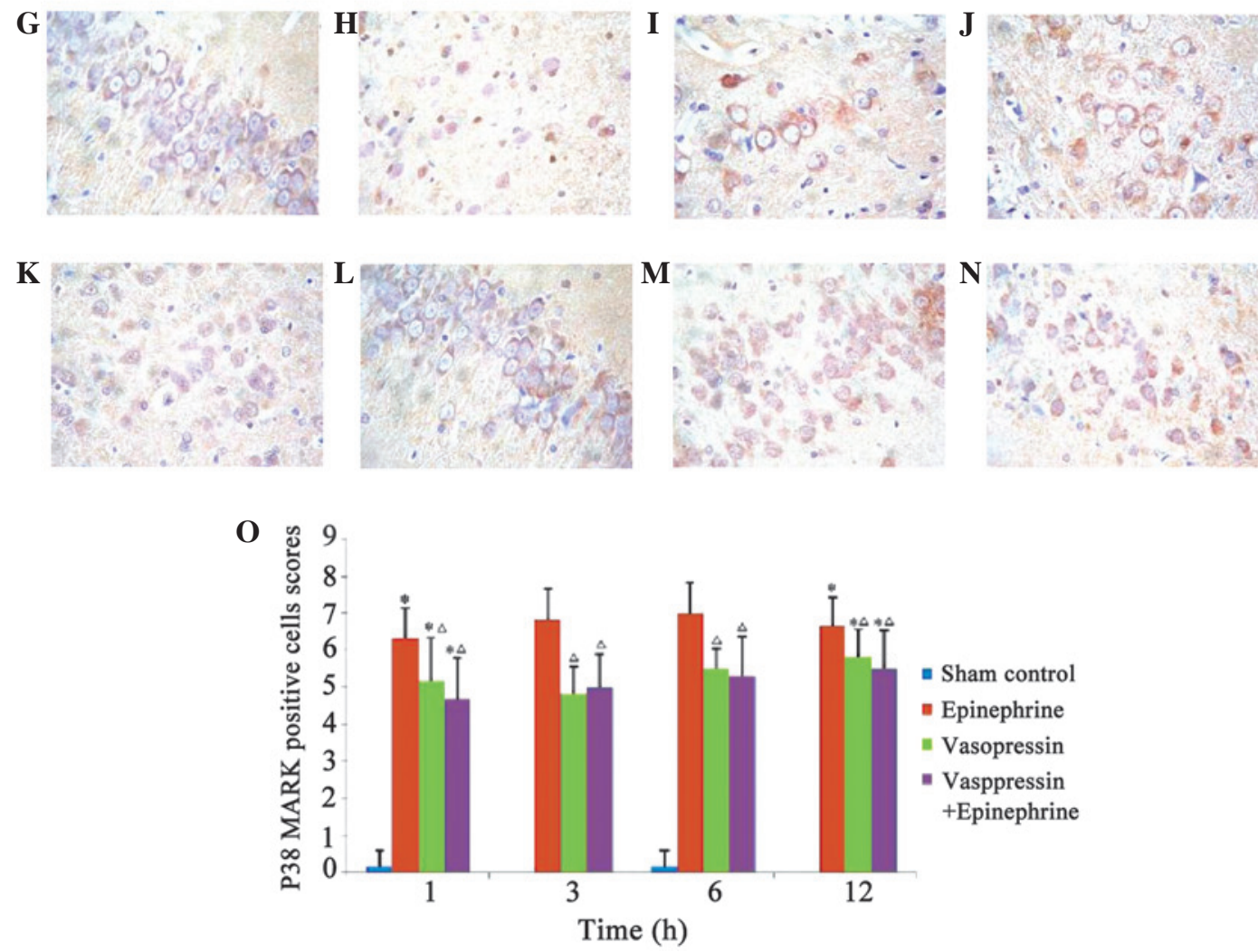

Figure 3. Immunohistochemical analysis of p38 mitogen-activated protein kinase (MAPK) expression following asphyxial cardiac arrest. (A and B) Immunohistochemical staining in the sham control group presented with a small quantity of positive p38 MAPK staining in the cytoplasm and nuclei. (C-F) p38 MAPK expression in the hippocampus of rats treated with epinephrine. Positive p38 MAPK staining was detected in the cell nucleus and the cytoplasm at (C) 1, (D) 3, (E) 6 and (F) $12 \mathrm{~h}$ following the restoration of spontaneous circulation (ROSC). (G-J) p38 MAPK expression in the hippocampus of rats treated with vasopressin. Positive p38 MAPK staining was detected in the nuclei and the cytoplasm at (G) 1, (H) 3, (I) 6 and (J) $12 \mathrm{~h}$ following ROSC. (K-N) p38 MAPK expression in the hippocampus of rats treated with vasopressin plus epinephrine. Positive p38 MAPK staining was detected predominantly in the cytoplasm and nuclei of neurons at (K) 1, (L) 3, (M) 6 and (N) $12 \mathrm{~h}$ following ROSC. (O) Positive staining scores for p38 MAPK across all treatments

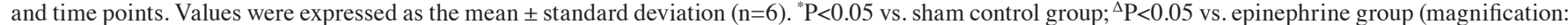
A and $\mathrm{C}-\mathrm{N}, \mathrm{x} 400 ; \mathrm{B}, \mathrm{x} 200)$.

may be activated by hypoxia and ischemia/reperfusion injury. Treatment with epinephrine alone did not significantly affect the expression levels of p38 MAPK following ROSC; however, vasopressin alone or in combination with epinephrine significantly reduced p38 MAPK expression levels in the hippocampus, as assessed by immunohistochemistry.

The fate of cerebral cells under anoxic conditions or in ischemia/reperfusion injury is partly determined by proteins of the apoptotic cascade, including NF- $\mathrm{kB}$ (10). Inhibition of the apoptotic pathway activated by NF- $\mathrm{KB}$ may attenuate cerebral injury (10). In addition, NF- $\mathrm{kB}$ is a critical transcription factor involved in inflammatory mediator induction; therefore, inhibition of NF- $\mathrm{KB}$ signaling may inhibit the expression of inflammatory mediators and attenuate subsequent inflammatory injury (10).

The results from the present study indicated that NF- $\mathrm{KB}$ p65 expression was elevated following asphyxial CA and ROSC, suggesting that these pathways are involved in hippocampal injury. It was also observed that combination therapy with vasopressin and epinephrine reduced NF- $\mathrm{KB}$ p65 expression levels to a greater extent than treatment with epinephrine alone. In addition, the results of the present study suggested that vasopressin was able to improve the post-ROSC outcome by suppressing apoptosis. 
A
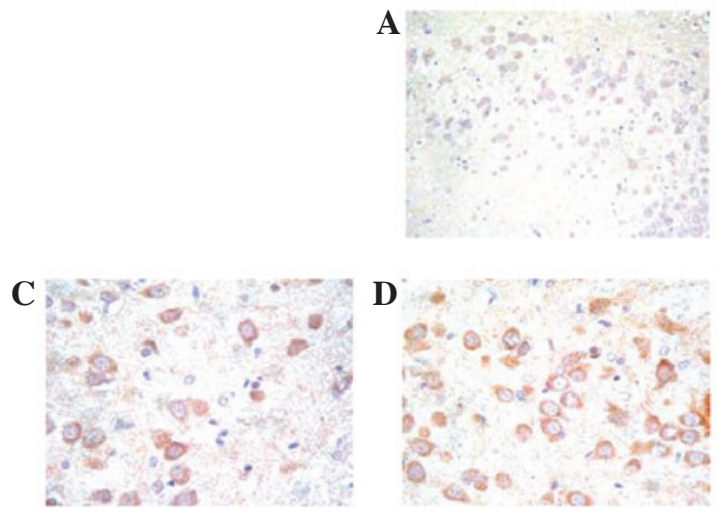

$\mathbf{H}$
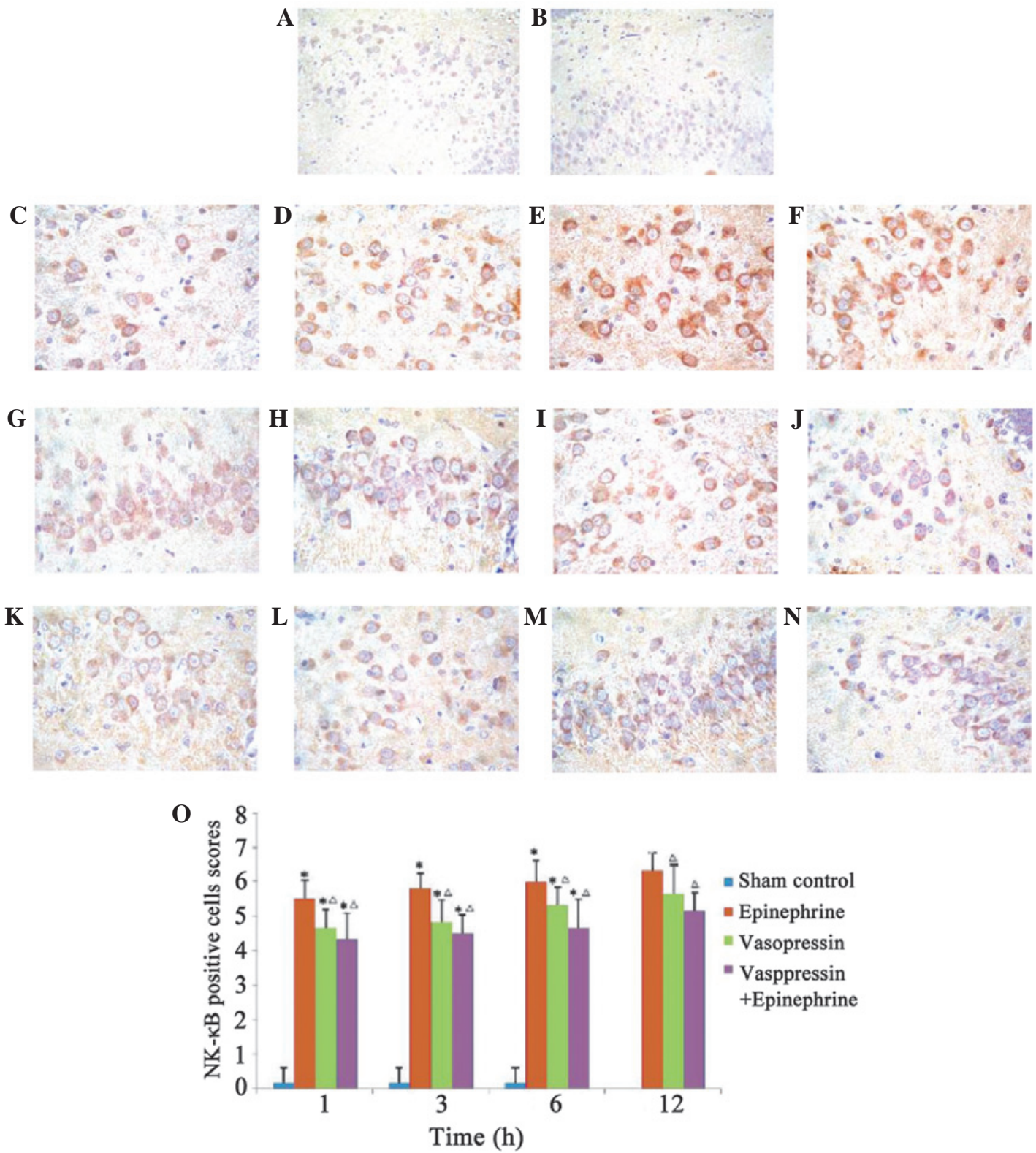

Figure 4. Immunohistochemical analysis of nuclear factor- $\mathrm{kB}$ (NF- $\mathrm{kB}$ ) p65 expression following asphyxial cardiac arrest. (A and B) Immunohistochemical staining in the sham control group showed very little positive NF-kB p65 staining in the cytoplasm and nuclei. (C-F) NF-kB p65 expression in the hippocampus of rats treated with epinephrine. Positive NF-kB p65 staining was detected in the cell nucleus and the cytoplasm at (C) 1, (D) 3, (E) 6 and (F) $12 \mathrm{~h}$ following restoration of spontaneous circulation (ROSC). (G-J) NF- $\mathrm{kB}$ p65 expression in the hippocampus of rats treated with vasopressin. Positive NF- $\mathrm{kB}$ p65 staining was detected in the nuclei and the cytoplasm at $(\mathrm{G}) 1,(\mathrm{H}) 3$, (I) 6 and $(\mathrm{J}) 12 \mathrm{~h}$ following ROSC. (K-N) NF-kB p65 expression in the hippocampus of rats treated with vasopressin plus epinephrine. Positive NF- $\mathrm{kB}$ p 65 staining was detected predominantly in the cytoplasm and nuclei of neurons in the vasopressin plus epinephrine group at (K) 1, (L) 3, (M) 6 and (N) $12 \mathrm{~h}$ following ROSC. (O) Positive staining scores for NF- $\mathrm{kB}$ p65 across all treatments and time points. Values are expressed as the mean \pm standard deviation $(\mathrm{n}=6)$. ${ }^{*} \mathrm{P}<0.05$ vs. sham control group; ${ }^{\wedge} \mathrm{P}<0.05$ vs. epinephrine group (magnification: $\mathrm{A}$ and $\mathrm{C}-\mathrm{N}, \mathrm{x} 400 ; \mathrm{B}, \mathrm{x} 200$ ).

One limitation of the present study was the 12-h observation period following resuscitation. Necrosis is difficult to detect within $12 \mathrm{~h}$ following ROSC; an observation time of $\geq 96 \mathrm{~h}$ would more accurately indicate hippocampal changes. In addition, the present study did not record neurological deficit scale scores, which would permit the analysis of the correlation between the extent of hippocampal injury and neurological function. Furthermore, there was no measurement of cerebral blood flow during CPR and in the post-resuscitation period; cerebral blood flow would indicate the mechanism by which vasopressin exerts its protective effects. Finally, no rats were administered a vehicle substance; vehicle controls would be required in order to accurately compare the effects of epineph- rine and vasopressin with the effects observed following no pharmacological intervention. Future studies are required to address these issues.

In conclusion, the present study demonstrated that the administration of vasopressin efficiently attenuated hippocampal injury during ROSC in a rat model of asphyxial CA, and was superior compared to treatment with epinephrine. In comparison to treatment with epinephrine, vasopressin alone and in combination with epinephrine was associated with more frequent ROSC and a more effective attenuation of hippocampal injury. The neuroprotective effects observed in the present study may be attributed to the inhibition of $\mathrm{p} 38$ MAPK and NF- $\mathrm{B}$ expression. Additional studies specifically 
addressing the effects of vasopressin on neurological outcomes are required in order to determine the mechanisms by which vasopressin reduces hippocampal injury.

\section{Acknowledgements}

The present study was supported by a research grant from the National Natural Science Foundation of China (grant no. 81471830).

\section{References}

1. Gueugniaud PY, David JS, Chanzy E, Hubert H, Dubien PY, Mauriaucourt P, Bragança C, Billères X, Clotteau-Lambert MP, Fuster P, et al: Vasopressin and epinephrine vs. epinephrine alone in cardiopulmonary resuscitation. N Engl J Med 359: 21-30, 2008

2. Mentzelopoulos SD, Zakynthinos SG, Tzoufi M, Katsios N, Papastylianou A, Gkisioti S, Stathopoulos A, Kollintza A, Stamataki E and Roussos C: Vasopressin, epinephrine and corticosteroids for in-hospital cardiac arrest. Arch Intern Med 169: 15-24, 2009.

3. Rudiger A, Tobler D and Estlinbaum W: Frequency and outcome of in-hospital resuscitation outside the ICU-setting. Swiss Med Wkly 134: 59-62, 2004

4. Stiell IG, Wells GA, Field B, Spaite DW, Nesbitt LP, De Maio VJ, Nichol G, Cousineau D, Blackburn J, Munkley D, et al: Advanced cardiac life support in out-of-hospital cardiac arrest. N Engl J Med 351: 647-656, 2004.

5. Girotra S, Nallamothu BK, Spertus JA, Li Y, Krumholz HM and Chan PS; American Heart Association Get with the Guidelines-Resuscitation Investigators: Trends in survival after in-hospital cardiac arrest. N Engl J Med 367: 1912-1920, 2012.

6. Luo CL, Li QQ, Chen XP, Zhang XM, Li LL, Li BX, Zhao ZQ and Tao LY: Lipoxin A4 attenuates brain damage and downregulates the production of pro-inflammatory cytokines and phosphorylated mitogen-activated protein kinases in a mouse model of traumatic brain injury. Brain Res 1502: 1-10, 2013.

7. Maroney AC, Glicksman MA, Basma AN, Walton KM, Knight E Jr, Murphy CA, Bartlett BA, Finn JP, Angeles T, Matsuda Y, et al: Motoneuron apoptosis is blocked by CEP-1347 (KT 7515), a novel inhibitor of the JNK signaling pathway. J Neurosci 18: 104-111, 1998.

8. Barone FC, Irving EA, Ray AM, Lee JC, Kassis S, Kumar S, Badger AM, Legos JJ, Erhardt JA, Ohlstein EH, et al: Inhibition of p38 mitogen-activated protein kinase provides neuroprotection in cerebral focal ischemia. Med Res Rev 21: 129-145, 2001.

9. Yasuda S, Sugiura H, Tanaka H, Takigami S and Yamagata K: p38 MAP kinase inhibitors as potential therapeutic drugs for neural diseases. Cent Nerv Syst Agents Med Chem 11: 45-59, 2011.

10. van Loo G, De Lorenzi R, Schmidt H, Huth M, Mildner A, Schmidt-Supprian M, Lassmann H, Prinz MR and Pasparakis M Inhibition of transcription factor NF-kappaB in the central nervous system ameliorates autoimmune encephalomyelitis in mice. Nat Immunol 7: 954-961, 2006.

11. Niu YL, Zhang WJ, Wu P, Liu B, Sun GT, Yu DM and Deng JB: Expression of the apoptosis-related proteins caspase-3 and NF-kappaB in the hippocampus of Tg2576 mice. Neurosci Bull 26: 37-46, 2010.

12. Xu L, Zhan Y, Wang Y, Feuerstein GZ and Wang X: Recombinant adenoviral expression of dominant negative IkappaBalpha protects brain from cerebral ischemic injury. Biochem Biophys Res Commun 299: 14-17, 2002.

13. van der Kooij MA, Nijboer CH, Ohl F, Groenendaal F, Heijnen CJ, van Bel F and Kavelaars A: NF-kappaB inhibition after neonatal cerebral hypoxia-ischemia improves long-term motor and cognitive outcome in rats. Neurobiol Dis 38: 266-272, 2010.
14. Wang JY, Shen J, Gao Q, Ye ZG, Yang SY, Liang HW, Bruce IC, Luo BY and Xia Q: Ischemic postconditioning protects against global cerebral ischemia/reperfusion-induced injury in rats. Stroke 39: 983-990, 2008.

15. Callaway CW: Epinephrine for cardiac arrest. Curr Opin Cardiol 28: 36-42, 2013

16. Nakahara S, Tomio J, Nishida M, Morimura N, Ichikawa M and Sakamoto T: Association between timing of epinephrine administration and intact neurologic survival following out-of-hospital cardiac arrest in Japan: A population-based prospective observational study. Acad Emerg Med 19: 782-792, 2012.

17. Nakahara S, Tomio J, Takahashi H, Ichikawa M, Nishida M, Morimura N and Sakamoto T: Evaluation of pre-hospital administration of adrenaline (epinephrine) by emergency medical services for patients with out of hospital cardiac arrest in Japan: Controlled propensity matched retrospective cohort study. BMJ 347: f6829, 2013.

18. Lurie KG, Voelckel WG, Iskos DN, McKnite SH, Zielinski TM, Sugiyama A, Wenzel V, Benditt D and Lindner KH: Combination drug therapy with vasopressin, adrenaline (epinephrine) and nitroglycerin improves vital organ blood flow in a porcine model of ventricular fibrillation. Resuscitation 54: 187-194, 2002.

19. Aoyagi T,Koshimizu TA and Tanoue A: Vasopressin regulation of blood pressure and volume: Findings from Vla receptor-deficient mice. Kidney Int 76: 1035-1039, 2009.

20. Mayr VD, Wenzel V, Voelckel WG, Krismer AC, Mueller T, Lurie KG and Lindner KH: Developing a vasopressor combination in a pig model of adult asphyxial cardiac arrest. Circulation 104: 1651-1656, 2001.

21. Stadlbauer KH, Wagner-Berger HG, Wenzel V, Voelckel WG, Krismer AC, Klima G, Rheinberger K, Pechlaner S, Mayr VD and Lindner KH: Survival with full neurologic recovery after prolonged cardiopulmonary resuscitation with a combination of vasopressin and epinephrine in pigs. Anesth Analg 96: 1743-1749, 2003.

22. Varvarousi G, Johnson EO, Goulas S, Agrogiannis G, Valsamakis N, Perrea D, Stefanadis C, Papadimitriou L and Xanthos T: Combination pharmacotherapy improves neurological outcome after asphyxial cardiac arrest. Resuscitation 83: $527-532,2012$

23. Kono S, Bito H, Suzuki A, Obata Y, Igarashi H and Sato S: Vasopressin and epinephrine are equally effective for CPR in a rat asphyxia model. Resuscitation 52: 215-219, 2002.

24. Chen MH, Xie L, Liu TW, Song FQ and He T: Naloxone and epinephrine are equally effective for cardiopulmonary resuscitation in a rat asphyxia model. Acta Anaesthesiol Scand 50: 1125-1130, 2006.

25. Chen MH, Liu TW, Xie L, Song FQ and He T: Does naloxone alone increase resuscitation rate during cardiopulmonary resuscitation in a rat asphyxia model? Am J Emerg Med 24: 567-572, 2006.

26. Remmele W and Stegner HE: Recommendation for uniform definition of an immunoreactive score (IRS) for immunohistochemical estrogen receptor detection (ER-ICA) in breast cancer tissue. Pathologe 8: 138-140, 1987 (In German).

27. Lim C, Alexander MP, LaFleche G, Schnyer DM and Verfaellie M: The neurological and cognitive sequelae of cardiac arrest. Neurology 63: 1774-1778, 2004.

28. Wenzel V, Linder KH, Augenstein S, Prengel AW and Strohmenger HU: Vasopressin combined with epinephrine decreases cerebral perfusion compared with vasopressin alone during cardiopulmonary resuscitation in pigs. Stroke 29: 1462-1467; Discussion 1467-1468, 1998.

29. Philpott KL and Facci L: MAP kinase pathways in neuronal cell death. CNS Neurol Disord Drug Targets 7: 83-97, 2008.

30. Sugino T, Nozaki K, Takagi Y, Hattori I, Hashimoto N, Moriguchi T and Nishida E: Activation of mitogen-activated protein kinases after transient forebrain ischemia in gerbil hippocampus. J Neurosci 20: 4506-4514, 2000. 